\title{
Condicionantes institucionales que influyen en la utilización del examen clínico de mama
}

0 felia Poblano-Verástegui, M en $C_{,}^{(1)}$ Juan Guillermo Figueroa-Perea, M en C, ${ }^{(2)}$ Lizbeth López-Carrillo, Dr Ph.(1)

Poblano-Verástegui O, Figueroa-Perea JG, López-Carrillo L. Condicionantes institucionales que influyen en la utilización del examen clínico de mama. Salud Publica Mex 2004;46:294-305. El texto completo en inglés de este artículo está disponible en: http://www.insp.mx/salud/index.html

\section{Resumen}

Objetivo. Identificar los condicionantes de la utilización del examen clínico de mama, la relación entre ellos y el ejercicio médico institucional. Material y métodos Entre 1996 y 1997 se hizo un estudio cualitativo en unidades médicas del Instituto Mexicano del Seguro Social y de la Secretaría de Salud. Se realizaron ocho grupos focales: cuatro con mujeres usuarias y cuatro con profesionales de la salud. Participaron 47 usuarias y 29 médicos y enfermeras. La información se interpretó con base en la capacidad de respuesta organizacional a las expectativas de la usuaria, el poder/saber en la organización y el ejercicio médico institucional, a la luz de laTeoría Fundamentada. Resultados La demanda estuvo condicionada por la percepción de mala calidad de la atención, falta de confianza en el médico y aspectos organizacionales. En la oferta, los médicos varones mostraron desinterés y temor a realizar el examen. Las médicas parecieron interesadas y las usuarias las aceptaron. Conclusiones. Existen barreras psicológicas, culturales, sociales e institucionales en el acceso y utilización del examen clínico de mama. La identificación de estas barreras y su origen pueden apoyar el desarrollo de acciones para mejorar la relación médico-paciente. El texto completo en inglés de este artículo está disponible en:http://www.insp.mx/ salud/index.html

Palabras clave: examen clínico de mama; relación médicopaciente; calidad de la atención médica; utilización de exámenes clínicos; grupos focales; M éxico

\author{
Poblano-Verástegui O, Figueroa-Perea JG, López-Carrillo L. \\ Institutional factors contributing \\ to the utilization of breast clinical examination. \\ Salud Publica Mex 2004;46:294-305. \\ The English version of this paper \\ is available at: http://www.insp.mx/salud/index.html
}

\begin{abstract}
A bstract
O bjective.To identify factors associated with utilization of breast clinical examination (BCE) and their relationship with institutio nal medical practice. Material and Methods.This is a qualitative study conducted between 1996 and 1997 in medical units of Instituto Mexicano del Seguro Social (Mexican Institute of Social Security) and Secretaría de Salud (Ministry of $\mathrm{H}$ ealth). Eight focus groups were included: four groups of female users and four groups of health professionals; in total, 47 users and 29 physicians and nurses participated. Interpretations of information were based on the organizational ability to respond to the user's expectations, "ability/knowledge" within the organization and the institutional medical practice, at the light of the Grounded Theory. Results. Service demand was conditioned on the perception of poor quality of care, lack of trust in physicians, and organizational aspects.W hen providing care, male physicians were not interested and felt uneasy about performing the $B C E$. Female physicians seemed more interested and were well accepted by users. Conclusions. Psychological, cultural, social, and institutional barriers exist in the access and utilization of BCE. Identifying these barriers and their origins could support the development of actions to improve the physician-patient relationship.The English version of this paper is available at: $h t t p: / / w w w . i n s p . m x / s a l u d /$ index.html
\end{abstract}

Key words: breast clinical examination; physician-patient relationship; quality of medical attention; utilization of clinical examinations; focal groups; Mexico

Este trabajo fue financiado con fondos del Consejo N acional de Ciencia y Tecnología de México (Proyecto Ref. 1371P-M9506. 0 ctubre de 1996).

(1) Instituto $\mathrm{N}$ acional de Salud Pública. Cuernavaca, Morelos, México.

(2) El Colegio de México. México, DF, México.

Fecha de recibido: 27 de mayo de 2003 • Fecha de aprobado: 1 de junio de 2004

Solicitud de sobretiros: 0 felia Poblano Verástegui. Dirección de O rganización en Sistemas de Salud, Centro de Investigación en Sistemas de Salud, Instituto N acional de Salud Pública. Avenida Universidad 655, colonia Santa María A huacatitlán, 62518 Cuernavaca, Morelos, México. Correo electrónico:opoblano@ correo.insp.mx 
D urante los últimos 10 años la incidencia del cáncer de mama (CM) ha aumentado en todo el mundo y México no es la excepción. A escala mundial, se estima que cada año se diagnostican aproximadamente un millón de casos y mueren por esta causa unas 372 mil mujeres. ${ }^{1}$ En Estados Unidos de América (EUA) este cáncer es la segunda causa de muerte en las mujeres y su incremento es consistente. Entre 1990 y 2000 se documentó un aumento anual de $1.4 \%{ }^{2,3}$ En América Latina, Uruguay y Chile reportan un problema similar, aunque aparentemente más grave: en el mismo periodo, el último país tuvo un incremento anual de $3.7 \%{ }^{3}$ En México la incidencia de CM ha aumentado lenta y consistentemente. En 1982 su tasa fue de 1.8 por 100000 mujeres mayores de 25 años de edad, en 1989 se incrementó a 2.9 y para 1997 la tasa alcanzó una cifra de 3.9, lo que representa un incremento de $116 \%$ en 15 años. ${ }^{1,3}$

La mortalidad por este tipo de cáncer, por su parte, aunque ha mostrado incremento, no alcanza los niveles de la incidencia. En México se estima que el promedio anual de incremento es de $1 \%$, aunque en algunos años ha sido mayor. ${ }^{3}$ En 2001 la tasa nacional de mortalidad fue de 14.7 por 100000 mujeres mayores de 25 años de edad. Las variaciones al interior del país oscilan de tasas inferiores a 10 (Campeche, Chiapas, Oaxaca, Quintana Roo, Guerrero) hasta superiores a 20 por 100000 (Sonora, Coahuila y Distrito Federal). ${ }^{3}$ Se ha estimado que más de $80 \%$ de los casos de cáncer que se detectan en México están en etapa avanzada, porque la detección temprana es poco frecuente. ${ }^{4}$

La Organización Mundial de la Salud (OMS) refiere a la detección temprana del cáncer como la única medida cuyo resultado se refleja en un incremento del tiempo de sobrevida y la reducción de la mortalidad por este padecimiento. ${ }^{5,6}$ Las estrategias son la prevención primaria y la secundaria. La primera, que modifica el riesgo, tiene un impacto limitado, ya que la mayoría de los factores para este cáncer no son modificables; así, los esfuerzos del sistema de salud deben enfocarse en la prevención secundaria (o detección temprana), la cual permite un pronóstico favorable en el tratamiento de esta enfermedad. ${ }^{6}$ En los países desarrollados se han aplicado programas poblacionales que promueven el autoexamen y la exploración clínica de mama; en algunos, como EUA y Canadá, se ha promovido activamente la mastografía.

La estrategia de prevención en México está señalada en la Norma Oficial Mexicana (NOM) 041-SSA22002 para la Prevención, Diagnóstico, Tratamiento, Control y Vigilancia Epidemiológica del Cáncer de Mama. ${ }^{7}$ La actualización publicada en el Diario Oficial de la Federación el 17 de septiembre de 2003 establece que: a) todas las mujeres deben realizarse mensualmente el autoexamen de mama (AEM) después de la menarca, y que es responsabilidad del prestador de servicios la enseñanza de la técnica de autoexploración; b) para las mujeres mayores de 25 años de edad se recomienda el examen clínico de mama (ECM) cada año, que deberá realizar el médico o la enfermera capacitada, en todas las unidades médicas del sector salud, previa autorización de la interesada, y c) la mastografía debe practicarse anual o bianualmente a las mujeres mayores de 40 años de edad con dos o más factores de riesgo, y anualmente a toda mujer mayor de 50 años, por indicación médica y con autorización de la interesada, de existir el recurso. Agrega que el ultrasonido es un recurso a utilizar en el caso de que se detecte patología mamaria en la exploración clínica. Además, la realización de la mastografía/ultrasonido en las unidades médicas de la Secretaría de Salud (SSA) no debe depender de la capacidad de pago. ${ }^{7}$

La demanda de un servicio médico por parte del usuario es resultado de la combinación de múltiples factores, entre ellos la disponibilidad y la aceptación del servicio, la percepción de la necesidad y la evaluación que el usuario hace de la calidad del mismo., Siempre que se hace un juicio sobre la cantidad en un servicio, está implícito un juicio sobre su calidad. ${ }^{10} \mathrm{Un}$ servicio con baja utilización tiene barreras de acceso, de demanda, de oferta, de percepción de calidad o todas ellas. De acuerdo con Donabedian, es necesario evaluar la calidad tanto en su componente técnico como en el interpersonal. Las dos dimensiones están íntimamente relacionadas y son interdependientes. ${ }^{11}$

Los participantes en el ECM son la usuaria y el profesional de la salud. Ella representa a una población en riesgo, y el prestador de servicios al sistema de salud. Las barreras pueden ser psicológicas, sociales, organizacionales o económicas, entre otras. ${ }^{12}$

En los últimos años se ha mostrado que la mujer, por su condición de género, enfrenta situaciones especiales en el uso de servicios de salud. Esto empeora por factores socioeconómicos, la posición que ocupa en la fuerza laboral (de beneficiarias y no derechohabientes), la escolaridad, el origen étnico, ${ }^{13-15}$ y la edad $^{16,17}$ son motivo para recibir calidad diferencial en la atención médica. En México, una encuesta reciente enfocada en la evaluación de la capacidad de respuesta del sistema de salud a las expectativas no médicas de los usuarios mostró que las mujeres perciben deficiencias de la calidad en la comunicación (78\%), en las instalaciones ( $74 \%$ ) y en la capacidad de elección (76\%) en relación con el profesional médico y con el servicio de salud. ${ }^{18}$ 
El intercambio interpersonal y el significado implícito en el ECM deben ser analizados desde lo social, los escenarios y contextos de cada participante, hasta sus contenidos cognoscitivos. Este es el campo de la investigación cualitativa, donde al explorar a profundidad los testimonios de los entrevistados es posible identificar los elementos que se heredan a través de la estructura social de los individuos o que se van conformando y se constituyen dominantes. ${ }^{19}$

Después de más de 25 años del programa de prevención de $\mathrm{CM}$ se desconocen sus resultados y su aplicación real. La disponibilidad y calidad técnica en el uso de los mastógrafos en el sistema de salud no permiten plantear que la mastografía pueda funcionar como una alternativa accesible de detección oportuna. ${ }^{20} \mathrm{El} \mathrm{ECM}$ está al alcance prácticamente de toda la población femenina, ya que puede practicarlo cualquier médico general o enfermera capacitada. No obstante, su utilización es baja. En una muestra de 1478 mujeres derechohabientes de la seguridad social y de la Secretaría de Salud se estimó una prevalencia de práctica del ECM de $24.4 \% .{ }^{21}$ En 2001, las estadísticas oficiales de servicios registraron 3543227 detecciones en cáncer de mama, sin especificar cuántos se debieron a ECM, mastografías y ultrasonidos de mama. ${ }^{22}$ En México, la población en riesgo, mujeres mayores de 25 años de edad, son 21825067 (44.51\% del total de la población femenina en el país) según el censo de 2000. ${ }^{23}$ Entonces, la cobertura real de la detección es mínima; sólo $7.22 \%$ de las mujeres en riesgo accedió a la detección oportuna del CM durante 2001.

Este trabajo buscó identificar algunos de los condicionantes institucionales -desde la organización y forma de realizar el trabajo- y socioculturales, que influyen en la utilización del ECM, ya que actúan sobre la dinámica de la relación médico-paciente en el marco de las instituciones de salud. Aunque los datos se recopilaron entre 1996 y 1997 se consideran válidos, ya que las condiciones en las cuales se otorga la atención dentro de las instituciones de salud en México no han cambiado sustancialmente. Además, el tiempo que requieren los cambios sociales para que éstos sean observables es prolongado, por lo que una modificación en la concepción de los papeles tradicionalmente asignados a los principales actores que participan en esta investigación seguramente tomará décadas.

\section{Material y métodos}

Entre 1996 y 1997 fueron estudiados cuatro grupos focales formados con usuarias de servicios de salud públicos en la Ciudad de México. Dos de estos grupos eran del Instituto Mexicano del Seguro Social (IMSS) y dos de la Secretaría de Salud del Distrito Federal. Las usuarias fueron invitadas en forma abierta, acudieron voluntariamente y se dividieron en dos grupos: menores y mayores de 40 años de edad. Se crearon estas categorías debido a la diferencia de riesgo que se ha documentado para el CM, además de suponer concepciones diferentes en la percepción del cuerpo y en el acto médico derivadas de la pertenencia a una generación diferente. A todas se les aplicó un cuestionario de identificación que contenía los datos sociodemográficos relevantes, los antecedentes personales patológicos y los de riesgo de $\mathrm{CM}$.

Además, en cada institución se realizaron dos grupos focales con personal de salud de las dos instituciones mencionadas, uno con médicos y el otro con médicas y enfermeras. A todos se les aplicó un cuestionario que contenía preguntas acerca de su identificación, antecedentes académicos, especialidad, años de práctica institucional y privada; también incluyó preguntas acerca del conocimiento de la NOM-014-SSA21994 (vigente en ese momento), y si tenían algún conocimiento de los resultados de encuestas enfocadas en evaluar la satisfacción de los usuarios, generalmente en el marco de encuestas nacionales.

En el grupo se trabajó con una guía de entrevista diferente para cada tipo de participante. Los grupos fueron conducidos por expertos en la técnica (un moderador y una moderadora). Se introducía un cuestionamiento para después "dejar hablar" a los participantes. Se concibió de manera similar a la libre asociación, a modo de cubrir el supuesto metodológico primordial: a través del discurso es posible identificar el contenido latente y subyacente del curso del pensamiento de los participantes, encontrar una estructura y un sentido en él. ${ }^{19}$

El análisis del contenido de las discusiones grupales se hizo siguiendo la sistematización sugerida por la Teoría Fundamentada. Esta teoría sostiene que la complejidad y variabilidad de los fenómenos sociales derivan de los significados preexistentes en las personas. De acuerdo con este modelo de interpretación de la realidad, las personas juegan el papel de actores que adoptan un papel activo al responder ante las situaciones de la vida diaria, situaciones a su vez definidas por el contexto en el que se presentan. De esta manera, la teoría afirma que las personas actúan con una intención (no necesariamente consciente) que se define y redefine constantemente como resultado de la interacción con el mundo. La interacción permanente evoluciona los procesos, pero el contexto (estructura) permanece relativamente estable. La relación entre el contexto (condiciones, situaciones), la acción (procesos) y las consecuencias, es constante. ${ }^{24,25}$ 
Las sesiones se grabaron y posteriormente se transcribieron. Las etapas de trabajo se resumen en: a) se revisó cada una de las transcripciones para generar códigos simples; $b$ ) se clasificaron las transcripciones con códigos abiertos; c) se generaron grupos de los códigos por ejes, conforme a su frecuencia y contenido, y d) se nombró a cada eje según su temática. Finalmente, se construyeron seis categorías analíticas desde donde se realizó la interpretación, a saber:

1. Género: autoestima y vivencia del cuerpo: los contenidos relacionados con pudor, vergüenza, pena y cohibición por mostrar el cuerpo y los senos, así como el temor a sufrir un abuso.

2. Percepción de la seducción: son los contenidos de sexualidad no explícita, de confusión, de duda, donde se mezclan las interpretaciones de la percepción propia y del "otro".

3. Enfermedad o sospecha de enfermedad: la presencia de síntomas, la negación de los mismos, los relatos de cáncer en la familia, el temor a conocer un diagnóstico.

4. Relación médico-paciente: aquellos contenidos derivados de la relación médico-paciente y que la usuaria identifica como facilitadores u obstáculos para la utilización del ECM, y que aunque se relacionan con aspectos de género, también contienen otras temáticas.

5. Institucionalización de la atención médica: contenidos que identifican lo que favorece u obstaculiza la utilización del ECM y que se deriva básicamente de la forma en como se organizan los servicios institucionales y de la consecuencia del ejercicio médico no independiente.

6. El papel de educador para la salud: remite a los contenidos en los cuales el médico aborda su participación en la educación para la salud, en especial la prevención del CM.

Tres de estas categorías se relacionaban con las condicionantes de género y se reportaron en otro documento; ${ }^{*}$ aquí se presentan únicamente las relacionadas con el entorno institucional del ejercicio de la medicina.

La calidad de la atención médica en la demanda, la oferta y, finalmente, la utilización está implícita en todas las categorías analíticas. Esto es, la compleja ex-

\footnotetext{
* Poblano-Verástegui O, Figueroa-Perea JG, López-Carrillo L. Algunas condicionantes de género en el acceso al examen clínico de mama. México, DF, México; 1999. Enviado para su publicación a Women \& Health.
}

periencia subjetiva detrás de cada proceso y de su interacción se manifiesta en los testimonios que corresponden a cada categoría, en algunos casos presentes como barreras y en otros como facilitadores de la utilización. El esquema de la interacción demanda-oferta para generar utilización se presenta en el cuadro I, donde se enumeran los antecedentes (variables que intervienen), tanto de la demanda como de la oferta y los elementos que entran en interacción durante la relación médico-paciente para que se dé la utilización del ECM.

Los datos sociodemográficos se analizaron a partir de frecuencias simples para describir las muestras participantes.

\section{Resultados}

El grupo de mujeres participantes en ambas instituciones fue de 47, 18 de 40 años o menos y 29 mayores de esa edad, con una media de casi 44 años. La mayoría de las mujeres tenía escolaridad primaria. Casi tres cuartas partes de las participantes se encontraban unidas. La descripción en detalle de las características de las usuarias participantes clasificadas por institución se presenta en el cuadro II.

En los antecedentes del CM que las mujeres informaron, una minoría tenía al menos un familiar cercano con CM (6), era nulípara (3) o padecía diabetes (1). Aproximadamente una sexta parte (7) había tenido su primer embarazo después de los 30 años de edad, había presentado la menopausia después de los 50 o había utilizado anticonceptivos orales. Poco más de una cuarta parte nunca había lactado o se consideraban obesas. El factor de riesgo presente en 20 de ellas era la menarca antes de los 12 años de edad. Sólo nueve de ellas acumulaban más de cuatro factores de riesgo a la vez.

El grupo del personal de salud participante constó de 17 médicos, 11 médicas y dos enfermeras. Las características detalladas por institución se presentan en el cuadro III. La media de edad del grupo completo fue de casi 42 años, mientras que la media de los médicos era casi cinco años inferior a la de las médicas; de igual forma, ellos acumulaban también menor experiencia institucional. Por el contrario, en ambas instituciones los médicos manifestaron tener consulta privada en mayor número que las médicas.

Otros datos no mostrados del personal de salud son: de la mitad del grupo con especialidad, la mayoría (10) la había cursado en el IMSS; la media de tiempo en experiencia institucional para todos fue de 10 años y los que ejercían práctica privada tenían una experiencia media igual. Dos médicas y un médico labo- 


\section{Cuadro I \\ Diagrama para el análisis del proceso de la atención en el examen clínico de mama. MÉxICO, DF, 1996-1997}

\begin{tabular}{|c|c|c|c|c|}
\hline \multicolumn{2}{|c|}{ Demanda } & \multirow{2}{*}{$\begin{array}{c}\text { Utilización } \\
\text { Atención médica }\end{array}$} & \multicolumn{2}{|c|}{ Oferta } \\
\hline Antecedentes (variables) & Elementos de interacción & & Elementos de interacción & Antecedentes (variables) \\
\hline $\begin{array}{l}\text { Características sociode- } \\
\text { mográficas: edad, educación, }\end{array}$ & Identidad de género & & Identidad de género & $\begin{array}{l}\text { Características sociodemográfi- } \\
\text { cas: sexo, edad, estado civil, es- }\end{array}$ \\
\hline & Relaciones con el poder & & Auto estima & colaridad \\
\hline $\begin{array}{l}\text { población económicamente } \\
\text { activa, nivel socioeconómico }\end{array}$ & $\begin{array}{l}\text { Tipo de institución donde soli- } \\
\text { cita el servicio }\end{array}$ & & $\begin{array}{l}\text { Relaciones con el poder y } \\
\text { forma de ejercer el poder }\end{array}$ & \\
\hline $\begin{array}{l}\text { Proceso de socialización de } \\
\text { género }\end{array}$ & $\begin{array}{l}\text { Educación para la salud, auto- } \\
\text { cuidado }\end{array}$ & $\begin{array}{l}\text { Examen clínico de } \\
\text { mama }\end{array}$ & $\begin{array}{l}\text { Lugar de poder que se ocu- } \\
\text { pa en el ejercicio profesional } \\
\text { institucional }\end{array}$ & $\begin{array}{l}\text { Proceso de socialización de gé- } \\
\text { nero }\end{array}$ \\
\hline $\begin{array}{l}\text { Características socio demo- } \\
\text { gráficas del esposo o compa- } \\
\text { ñero }\end{array}$ & $\begin{array}{l}\text { Conocimientos acerca del cán- } \\
\text { cer de mama } \\
\text { Saber popular }\end{array}$ & & $\begin{array}{l}\text { Conocimientos y saber mé- } \\
\text { dico }\end{array}$ & $\begin{array}{l}\text { "Deber ser" médico (identidad } \\
\text { profesional) }\end{array}$ \\
\hline $\begin{array}{l}\text { Experiencias propias o de } \\
\text { personas cercanas con el sis- } \\
\text { tema de salud y el cáncer en } \\
\text { especial }\end{array}$ & $\begin{array}{l}\text { Expectativas respecto a la cali- } \\
\text { dad del servicio, con base en } \\
\text { las experiencias previas, propias } \\
0 \text { ajenas }\end{array}$ & & $\begin{array}{l}\text { Institución donde se encuen- } \\
\text { tra trabajando } \\
N \text { ivel de satisfacción laboral }\end{array}$ & Formación médica o profesional \\
\hline
\end{tabular}

raban en más de una institución de salud del sector público. De las preguntas de conocimiento aplicadas sobre los criterios para ofrecer el ECM de acuerdo con la NOM, sólo tres respondieron adecuadamente. Los testimonios que se presentan se identifican mediante las siglas entre paréntesis, se señala la institución de salud, el número del grupo y el año; además de las siglas MA, para médicas, MO, para médicos y ENF, para enfermeras.

\section{Relación médico-paciente}

En el saber popular se ha venido reproduciendo una serie de ideas y valores sociales que obstaculizan la búsqueda activa de la mujer hacia la atención preventiva. Por una parte están las referencias valorativas en términos de que la mujer debe "ver por la salud de los demás" incluso antes de ver por la de ella misma; Franca Basaglia ${ }^{26}$ habla de un "ser para los otros", ya que el proceso de socialización de muchas mujeres las lleva a negarse a sí mismas, con el fin de dedicarse al cuidado de los demás. Por otra parte, esta situación se complica especialmente cuando los demás miembros de la familia (vecinos, amigos, red social) creen que no requiere esa atención. La mujer necesita tomar la decisión de emprender una acción y comúnmente se ve influida por los demás para hacerlo. Esta influencia puede ser un obstáculo aun mayor cuando su entorno familiar o social no ha tenido buenas experiencias con el sistema de salud. El contenido de este saber popular se basa en la experiencia de las personas cercanas a la usuaria con las instituciones de atención médica:

... quiero saber cómo cuidar mi cuerpo, quitarme esas malas ideas que me meten en mi casa, porque le digo que mi suegra me dice, no (acudas) que fulanito (el médico) me trató muy mal, y mira, no, es que ese viejo (el médico) es quien sabe qué y por eso, nunca vengo yo y no me acerco, pues no sé nada. (SSA1/1996).

... sobre todo a las jóvenes, vacilarlas y sobre todo los (médicos) del Seguro tienen fama de que toman mucho, inclusive dice un chico que es amigo de mi hijo, que trabaja en el Seguro, dice que a veces están bien crudos cuando están haciendo las operaciones, por eso luego les dejan (a los pacientes) la gasa, que esto, que pinzas, que quién sabe qué, porque están tomados... (SSA2/1996).

Esta participación trae a la discusión un contenido que, tanto en México como en otros países ${ }^{27}$ cada día aparece con mayor frecuencia en los medios de comunicación masiva y en la comunicación entre los miembros de la sociedad: la insatisfacción con el sistema de salud y con la actitud de los profesionales de la salud. De hecho, una manera de enfrentar al poder ejercido por la medicina hegemónica y las instituciones de salud es la devaluación de su figura, su papel y su actuación. 


\section{Cuadro II}

Características de las mujeres participantes en el ESTUDIO SOBRE EXAMEN CLÍNICO DE MAMA. MÉxıco, DF, 1996-1997

\begin{tabular}{|c|c|c|c|}
\hline Variables & $\begin{array}{l}\text { Secretaría } \\
\text { de Salud } \\
\text { (n) }\end{array}$ & $\begin{array}{c}\text { Instituto } \\
\text { M exicano del } \\
\text { Seguro Social } \\
\text { (n) }\end{array}$ & $\begin{array}{l}\text { Total } \\
\text { (n) }\end{array}$ \\
\hline Mujeres participantes & 25 & 22 & 47 \\
\hline \multicolumn{4}{|l|}{ Escolaridad } \\
\hline N inguna & 1 & 1 & 2 \\
\hline Primaria incompleta o completa & 13 & 7 & 20 \\
\hline Secundaria/carrera comercial & 9 & 8 & 17 \\
\hline Preparatoria o más & 2 & 6 & 8 \\
\hline \multicolumn{4}{|l|}{ Estado Civil } \\
\hline Unidas & 22 & 15 & 37 \\
\hline Solteras & 3 & 7 & 10 \\
\hline \multicolumn{4}{|l|}{0 cupación } \\
\hline Labores del hogar & 20 & 8 & 28 \\
\hline Trabajo remunerado & 5 & 12 & 17 \\
\hline Pensión o jubilación & 0 & 2 & 2 \\
\hline
\end{tabular}

Escolaridad del compañero

\begin{tabular}{llll} 
N inguna & 1 & & 1 \\
\hline Primaria incompleta o completa & 8 & 5 & 13 \\
\hline Secundaria/carrera comercial & 8 & 3 & 11 \\
\hline Preparatoria o más & 5 & 9 & 14 \\
\hline No sabe/no responde & 3 & 5 &
\end{tabular}

0 cupación del compañero

\begin{tabular}{lrrr} 
N inguna o pensión & 3 & 1 & 4 \\
\hline Empleado & 13 & 13 & 26 \\
\hline Independiente & 6 & 3 & 9 \\
\hline No sabe/no responde & 3 & &
\end{tabular}

Edad (en años)

\begin{tabular}{lcrr} 
Media & 40.7 & 46.8 & 43.6 \\
\hline DE & 13.4 & 12.3 & 13.1 \\
\hline Mín-máx & $23-82$ & $26-68$ & $23-82$
\end{tabular}

0 cupantes de la vivienda (además de ellas)

\begin{tabular}{llrr} 
Media & 5 & 4.9 & 4.9 \\
\hline DE & 2.9 & 3.6 & 2.2 \\
\hline Mín-máx & $2-14$ & $2-19$ & $2-19$
\end{tabular}

DE: desviación estándar

\section{Cuadro III}

Características de los médicos participantes en el ESTUDIO SOBRE EXAMEN CLÍNICO DE MAMA. MÉxICO, DF, 1996-1997

\begin{tabular}{|c|c|c|c|c|}
\hline \multicolumn{2}{|l|}{ Variables } & $\begin{array}{c}\text { Secretaría } \\
\text { de Salud } \\
\text { (n) } \\
16\end{array}$ & $\begin{array}{l}\text { Instituto } \\
\text { Mexicano del } \\
\text { Seguro Social } \\
\text { (n) } \\
14\end{array}$ & $\begin{array}{c}\text { Total } \\
\text { (n) } \\
30\end{array}$ \\
\hline \multirow[t]{3}{*}{ Participantes } & Médico & 8 & 9 & 17 \\
\hline & Médica & 8 & 3 & 11 \\
\hline & Enfermera & 0 & 2 & \\
\hline
\end{tabular}

Estado civil

\begin{tabular}{lrrr} 
Varones & & & \\
Unidos & 7 & 7 & 14 \\
\hline $\begin{array}{c}\text { Solteros } \\
\text { Mujeres }\end{array}$ & 2 & 1 & 3 \\
Unidas & 5 & 5 & 10 \\
\hline Solteras & 3 & 0 & 3
\end{tabular}

Facultad de origen

\begin{tabular}{lrrr} 
UNAM & 15 & 10 & 25 \\
\hline 0 tras & 1 & 4 & 5
\end{tabular}

Especialidad

\begin{tabular}{llll} 
Varones & & & \\
Sin especialidad & 5 & 3 & 8 \\
\hline Médico familiar & 1 & 5 & 6 \\
\hline Salud pública & 1 & 0 & 1 \\
\hline O tra especialidad & 1 & 1 & 2 \\
Mujeres & & & \\
Sin especialidad & 4 & 1 & 5 \\
\hline Médica familiar & 0 & 2 & 2 \\
\hline Salud pública & 0 & 2 & 2 \\
\hline Otra especialidad & 2 & 0 & 2 \\
\hline Enfermería & 2 & &
\end{tabular}

Práctica privada

\begin{tabular}{cccc}
$\begin{array}{c}\text { Varones } \\
\text { Sí }\end{array}$ & 5 & 4 & 9 \\
\hline $\begin{array}{c}\text { No } \\
\text { Mujeres } \\
\text { Sí }\end{array}$ & 3 & 5 & 8 \\
No & 2 & 2 & 4 \\
\hline & 6 & 3 & 9
\end{tabular}

Edad (en años)

\begin{tabular}{lcrr}
$\begin{array}{l}\text { Varones } \\
\text { Media }\end{array}$ & 40 & 39.3 & 39.6 \\
\hline DE & 5.85 & 11.4 & 8.9 \\
\hline $\begin{array}{c}\text { Mín-máx. } \\
\text { Mujeres } \\
\text { Media }\end{array}$ & $30-45$ & $23-57$ & $23-57$ \\
\hline DE & 46.2 & 41.4 & 44.3 \\
\hline Mín-máx. & 6.1 & 6.9 & 6.6 \\
\hline Mand & $39-58$ & $34-50$ & $34-58$
\end{tabular}

Experiencia institucional (años)

\begin{tabular}{cccr} 
Varones & & & \\
Media & 9.2 & 12.8 & 11.1 \\
\hline DE & 5.3 & 19.8 & 8.6 \\
\hline $\begin{array}{c}\text { Minn-máx. } \\
\text { Mujeres } \\
\text { Media }\end{array}$ & $2-17$ & $0-35$ & $0-35$ \\
\hline DE & 18.1 & 21.2 & 19.3 \\
\hline Mín-máx. & 4.7 & 10.2 & 7.0 \\
\hline & $12-27$ & $13-37$ & $12-37$
\end{tabular}

DE: desviación estándar

UN AM: Universidad N acional Autónoma de México 
Para las mujeres parece existir el dilema de asistir o no a los servicios médicos. Esperan mala atención, caracterizada por largos tiempos de espera, falta de información, maltrato y abuso y tener que aceptarlos -puesto que generalmente no hay alternativa- o definitivamente no atender su malestar. Es común que estas experiencias de maltrato se comuniquen a otros miembros de su familia, en especial femeninos. Pero difícilmente o nunca se exponen formalmente ante las autoridades de los servicios de salud. A las usuarias no les es posible confrontar al poder institucional, sobre todo cuando no tienen alternativa de elección de servicios médicos, ya sea por motivos económicos, condición de aseguramiento o ambos.

El contenido del discurso de las médicas avala lo dicho por las usuarias, saben del abuso del poder por parte de los médicos hacia las mujeres:

Sí, es frecuente que (las mujeres) se quejen de que los ginecólogos o médicos quieran hacerles el tacto por nada (MA-SSA/1996).

...desde que entra (la paciente) ya le está hablando de tú (el médico), y "a ver mamacita, quítate, ponte y hazte..." , eso ya la paciente lo siente como agresión de alguna manera (MA-SSA/1996).

...cuando vas a la consulta con una doctora, te revisa con más calma y el doctor no, nada más te sienta y ¿qué tiene? ... (SSA1/1996).

Para las médicas es claro que sus colegas varones fácilmente pueden actuar de forma autoritaria y agresiva hacia las mujeres, que su actitud frente a ellas es la de mostrar quién tiene el poder. Que el tuteo hacia la mujer de cualquier edad puede ser interpretado como una manifestación de ese poder y, además, indica superioridad y autoridad reconocida en el contexto médico institucional. Alguno de los médicos también identifica este tuteo, como se verá más adelante, como una falta de respeto hacia la usuaria.

Por el contrario, lo que actúa como un facilitador de la utilización del ECM, es la confianza construida a través de una relación médico-paciente prolongada, donde se ha establecido un vínculo de aceptación, respeto y confianza. En los grupos de médicos aparecieron los siguientes contenidos:

Son circunstancias que tienen mucho que ver con la sexualidad y el hecho de enfrentarse a una persona que es hombre ¿verdad? Como que se genera una barrera así terrible, que genera un impasse, yo creo que en ambos. Bueno, a mí me pasa con las señoras y por eso primero las voy midiendo para ver hasta dónde ellas son capaces realmente de poder aceptar la opinión que yo pudiera ofrecerles o inclusive la exploración (de senos), todo depende mucho de la facilidad con que se vaya dando esta relación médico-paciente (MO-SSA/1997). La forma en que tú te comportes con tus pacientes te va a dar o no la confianza. Si va a entrar una chica y ya estás como el zorro filoso y todo, aunque tenga 80 años, si desde que entra ya la estás (desvistiendo) con rayos infrarrojos ... pues, óyeme, este es un pelafustán, sencillamente no te tiene confianza y no te va a permitir nada; pero si desde que llega ve que realmente eres un profesional, que la estás respetando de verdad, porque si desde que entra tú le empiezas a tutear ..., para mí es una falta de respeto hacia la paciente, y de otra forma si tú ... le estás dando respeto, y ahí va todo ...(MO-IMSS/ 1996).

Las médicas comparten esta percepción con sus compañeros, aunque podría parecer muy obvio que ellas pueden ofrecer el ECM sin el temor de hacer sentir incomodidad a la paciente, identifican que esta confianza debe construirse lentamente, y que si no existen factores de riesgo o síntomas evidentes, el ofrecimiento puede esperar:

...establecer una buena relación médico-paciente, ya que tenemos la confianza, yo siempre me espero a tener este tipo de inspecciones (ECM) a la segunda o tercera consulta, a menos que detecte algunos factores de riesgo... (MA-IMSS/1997).

Los médicos también argumentan su falta de oferta hacia el ECM con base en factores sociales, psicológicos y culturales de la población. Señalan que socialmente existen barreras, se sugiere la falta de "educación médica", pero lo más relevante es el temor a la existencia actual de organismos oficiales auditores de la práctica médica, donde el paciente puede presentar su queja:

...existen factores socioculturales, como el compañero lo comenta, es verdad, la cultura de nuestra población nos bloquea en determinado momento, para que una persona vaya por iniciativa propia, que diga, "doctor yo vengo para que me explore las glándulas mamarias", a veces nosotros se los pedimos con algo de sutileza, pero tenemos desconfianza, sobre todo con este Comité que se formó (la Comisión Nacional de Arbitraje Médico*) ...(MO-SSA/1997).

* Cuando inició esta investigación, la Comisión Nacional de Arbitraje Médico (CONAMED) tenía dos años de creada. Actualmente es la organización que recibe y tramita las quejas e inconformidades de la mayoría de los usuarios de los servicios de salud del país. 
...actualmente, nosotros tenemos que estar ya más sensibilizados y a través de los medios de difusión al paciente se le ha concienciado acerca de sus derechos y lo que pueden hacer legalmente con nosotros, de que hay una Comisión de Arbitraje, pusieron en la Procuraduría de que hay un código penal de todo, hasta violaciones, etcétera, entonces esas técnicas (las del ECM) tienen que ser bastante rigurosas... (MO-IMSS/1996).

Los testimonios señalan que factores sociales, psicológicos y culturales se encuentran obstaculizando a las mujeres, a la vez que los mismos contenidos se encuentran en los médicos y de igual forma son obstáculos para el ofrecimiento del ECM.

Por lo mencionado tanto en el grupo de médicos como de médicas es más probable que las usuarias acepten el ECM si la relación médico-paciente se ha establecido desde varias sesiones atrás. El médico se ha ganado el respeto de la paciente y ésta a la vez se siente valorada y respetada por el profesional de la salud. La existencia o no de una buena relación médico-paciente puede ser una barrera para la utilización, hecho confirmado por otros autores. Esta barrera se ubica claramente en la demanda, pero además también obstaculiza la oferta, ya que en ninguno de los casos se menciona que sea la paciente quien solicita la exploración, sino que es el médico el que debe superar el probable rechazo a través de la construcción previa de la relación de colaboración con su paciente.

\section{Institucionalización de la atención médica}

Las instituciones públicas de salud tienen entre sus objetivos la promoción de la salud, esto es, la realización de actividades preventivas y detecciones que apoyan el mantenimiento de la salud. Entonces, muchas de sus acciones están enfocadas en la prevención. En el caso del CM, se debe ofrecer el ECM a toda mujer que acuda a consulta, según la NOM correspondiente y a través de médicos y enfermeras capacitadas. Las dos instituciones participantes han instrumentado el cumplimiento de la NOM de diferente manera. En el IMSS se designa formalmente a las enfermeras como responsables, a través de un módulo específico, para realizar detecciones de varias enfermedades, entre ellas el CM. La solicitud puede ser hecha por el médico o directamente por la paciente. En la SSA sólo los médicos tienen esta responsabilidad y se practica dentro de la consulta externa.

El hallazgo en las discusiones de los grupos fue que los médicos ofrecen poco o nunca el ECM. Cuando lo llegan a ofrecer, la usuaria generalmente acepta a pesar de todas las dudas y temores que surgen por la desconfianza en la institución y en el mismo médico. En la seguridad social, lo más frecuente es que los médicos señalen la disponibilidad del ECM, dando por hecho que las usuarias deben solicitarlo por propia iniciativa:

A mí la doctora me dijo que por qué no había ido; hasta me regañó: que no es necesario que nos estén diciendo que vayamos, que nosotros tenemos que ir (al módulo de prevención de $\mathrm{CaCu}$ y $\mathrm{CM}$ ), porque es por el bien de nosotras (SSA1/1996).

Sí, yo creo que sí porque la doctora tiene más autoridad y no pueden decir no, pero nosotras somos enfermeras y ya llegan enojadas y molestas... (ENF-IMSS/ 1997).

El nivel de responsabilidad con el autocuidado es el resultado de un proceso de educación y promoción de la salud al que hay que invertir tiempo y recursos, tanto desde la institución, como del personal de salud y del propio usuario. En CM sólo recientemente las instituciones de salud han difundido la importancia de la detección temprana.

En el IMSS, tal como señalan los documentos sobre organización de la atención, los médicos generalmente sólo indican a la paciente que acuda al módulo de detección, dejando en manos de las enfermeras la práctica del mismo. Sin embargo, las enfermeras se consideran con menos poder y conocimiento que los médicos. Esto genera tres situaciones: en una de ellas la paciente sí acude y se realiza el ECM, en la segunda acude pero cuestiona a la enfermera para finalmente no aceptar y, por último, no acude al módulo:

...llegó a pedirla (la exploración) pero también buscaba alguna justificación para decir no ...o sea, llegó a pedirla porque su médico se lo pidió, pero ya estando ahí (en el módulo) ya ellas (las pacientes) nos ven como... luego nos preguntan "¿es muy necesario que me lo hagan?, sí, me mandó el médico, pero yo no quiero", entonces (le decimos) "como guste, es necesario porque es muy importante que se hagan sus detecciones" y se niegan... (ENF-IMSS/1997).

Las enfermeras, quienes no tienen el mismo reconocimiento que los médicos dentro de la institución, refieren enfrentar las reacciones de las pacientes cuando no se ha llevado a cabo una buena comunicación y la paciente no tiene claridad respecto de su riesgo al CM ni la importancia de su prevención. Los médicos, tal como anteriormente las médicas, dicen que 
ante la presencia de síntomas ellos mismos realizan el ECM. Sin embargo, esto no es confirmado por las enfermeras:

...luego llegan las pacientes, "pues vengo a hacerme un examen de cáncer porque tengo una bolita", "¿ya pasó con su médico familiar a revisión?", "sí, pero me dijo que usted me lo tenía que hacer, el examen", entonces muchas veces ... como que ellas (las pacientes) no nos creen... (ENF-IMSS/1996).

El contenido de este testimonio, al igual que el introducido anteriormente en torno a la aceptación de las pacientes, cuando se señala que al médico "no se le puede decir que no", nuevamente sugiere que las enfermeras se ven a sí mismas disminuidas frente a la autoridad y el saber que, en las instituciones de salud, generalmente se deposita en el médico.

Las médicas explican las diferencias en la organización de la atención médica, y en especial la atención preventiva, entre las dos principales instituciones de salud del país. Consideran que en gran medida las fallas de la atención derivan de las políticas institucionales, y los pacientes se ven limitados por ellas. Como ejemplo se cita la imposibilidad de elegir libremente al médico del primer nivel de atención en el IMSS, acudir por la necesidad de cubrir los requisitos laborales a través de un documento oficial (incapacidad) o porque no se pueden cubrir los gastos adicionales si se busca atención médica en otro lugar, pero que los pacientes muestran mayor insatisfacción ante la atención otorgada. Todos estos factores influyen en la aceptación "obligada" de un servicio mientras que en la SSA el paciente elige si regresa con el mismo médico, no hay presión para aceptarlo:

...aquí la gente viene por gusto y no por obligación como en el Seguro, que a fuerza tiene que ir porque ahí le van a dar la medicina... la incapacidad, y aquí no, la gente viene realmente y a veces se identifica muy bien con cada uno de nosotros y hay gente que te sigue por muchos años y no te quiere cambiar y cosas así, y pienso que es por el buen trato... (MA-SSA/1996).

...los médicos (del IMSS) no hacen mucha educación para la salud, se dedican principalmente a su consulta para terminar... no les interesa otra cosa o al menos que tuvieran (las pacientes) algún problema, que les dijera la paciente que le duelen los senos o que tiene algún flujo, ...los médicos no tienen mucha información... (MA-SSA/1996).

Aunque históricamente los médicos de la seguridad social han contado con mayor prestigio en el sistema de salud, en relación con los médicos que atienden a población abierta, los hallazgos relacionados con acudir voluntariamente a la atención médica sugieren que los médicos de la seguridad social se encuentran más devaluados ante los usuarios que quienes laboran para la SSA, como ya se había mostrado en el testimonio donde se señala que "...los (médicos) del seguro tienen fama de que toman mucho..."

Los médicos, por su parte, también son críticos hacia la calidad en su trabajo y su responsabilidad con la salud de la población, tanto en la falta de actualización como de interés por su trabajo:

Podemos justificarnos por la carga de trabajo, pero la verdad yo creo que no hay justificación. La cosa es que ha habido poco interés por parte de nosotros, porque muchos de nosotros, pues a pesar de que hay programas, uno no tiene interés, cada quien se dedica a su trabajo, le dan una revisada (al paciente), le recetan penicilina y ya, no voy a decir que todos, pero algunos de nosotros no tenemos interés por desempeñar este Programa (detección de CM) (MO-SSA/1997).

Es importante hacer notar la forma en que se plantea "cada quien se dedica a su trabajo", como si ofrecer el ECM a la población femenina en riesgo no estuviera dentro de las actividades de su trabajo, fácilmente se identifica la devaluación de las acciones preventivas frente a las curativas. El médico trabaja "curando", no evitando la enfermedad. El médico es contratado por la institución de salud para "curar" enfermos no para "prevenir" o "enseñar" a la población a mantener la salud, esta actividad queda relegada para ser realizada por las enfermeras, profesionales de la salud con menos conocimiento, autoridad y poder.

\section{El papel de educador para la salud}

Cuando se solicita a los médicos que aporten ideas para mejorar la utilización del Programa de Prevención del $\mathrm{CM}$, concuerdan en que la base para que la mujer asuma su autocuidado es sensibilizar desde la escuela, desde los estudios primarios.

...actualmente es más fácil abrirle la vagina a una mujer que checarle el pecho, ¿por qué?, porque están más sensibilizadas y esto debe hacerse desde la primaria... (MO-SSA/1997).

Es una forma de reconocer que el trabajo preventivo de las instituciones de salud en torno al cáncer cérvico uterino ha sido apoyado por los contenidos relacionados con la salud de la mujer que se encuentran en los textos escolares, pero también es de señalar que, probablemente, sin esta labor desde otros ámbi- 
tos de la sociedad la toma de conciencia del autocuidado sería más difícil. Y, como una alternativa más, es una forma de limitar el papel de educador para la salud que tiene el médico, para depositar esta responsabilidad fuera del sector. Además, piensan que el éxito de la detección oportuna del CM debe ser apoyado con medios de comunicación masiva:

...si usted quiere algo que facilite de manera dramática la exploración de senos, es que se sensibilice a nivel nacional con medios masivos de comunicación. (MOSSA/1997).

Los médicos consideran que su labor como educadores es inferior a la que realiza la escuela e incluso los medios de comunicación masiva. Ninguno de ellos refirió que mediante su trabajo con los pacientes se hiciera posible modificar las actitudes generadas por la educación otorgada por la familia, o proporcionar a la usuaria suficiente información como para que comprenda la necesidad de realizar el ECM, el autoexamen o la mastografía. Los profesionales médicos piensan que estos aspectos pueden ser logrados a través de medios "modernos" no merced al trabajo de los profesionales de la salud.

Algunas de las mujeres participantes consideró que ellas están educando a sus hijas de manera diferente a como las educaron a ellas; intentan modificar la falta de información y conocimiento sobre el cuerpo y su fisiología, así como el papel pasivo que ellas tienen frente a su salud para favorecer el acercamiento de sus hijas a los servicios médicos preventivos, tal como lo señalan mujeres mayores de 40 años de edad:

...yo pienso que cuando mi hija llegue a una edad en la que se le pueda decir todo lo que se relaciona con su cuerpo, porque cuando yo era chamaca mi mamá en vez de decirme ... "sabes que llegamos a una edad en que empezamos con la menstruación", mi mamá nunca nos platicó de cómo cuidarnos ni qué nos iba a pasar en el cuerpo ... (IMSS2/1996).

...precisamente por lo mismo que nosotros vivimos nos ha servido de experiencia para abrirnos un poquito con nuestras hijas, enseñarlas y que vayan al médico, que las chequen... (SSA2/1996).

Como le digo si uno les dice sobre cuidarse las cosas cambian ... ellas (las hijas) sí van al seguro a revisión y a todo, pero yo como no tengo molestias nunca he ido, pero ellas sí van o sea que no es así como decía ella (se refiere a otra participante que habla de enseñar con el ejemplo), como ven que yo no voy ellas no van, no ... ellas sí van (SSA2/1996).

\section{Discusión}

En los testimonios seleccionados para mostrar las categorías analíticas relacionadas con la provisión de servicios médicos, las usuarias se refieren a algunos de los obstáculos que les impiden el acceso y la utilización del ECM, que derivan básicamente del ejercicio de la medicina institucional, la forma como está organizada y "la actitud del médico institucional" y del ejercicio del poder organizacional en la relación interpersonal médico-paciente; asimismo, a algunos aspectos que son identificados, en general, por las mujeres usuarias como mala calidad en la atención, y que se remiten a las fallas de proceso en el sistema de salud, que llegan a los medios de comunicación masiva como negligencias, iatrogenias o abusos médicos.

En el cuadro I, se sintetizan los factores encontrados en esta investigación, tanto los que actúan como facilitadores para la oferta como para la demanda. Para comprender por qué es más fácil la aparición de los obstáculos para la demanda y para la oferta, en las discusiones grupales, es necesario profundizar en los contenidos de estas explicaciones. Dichas explicaciones se remiten a lo que las mujeres han escuchado y han vivido en la sociedad y en la atención médica institucional. Ellas han introyectado los discursos populares y familiares, conjuntándolos y dándoles un nuevo significado. La posición de la mujer en la sociedad y, generalmente, el carácter de beneficiarias de la atención médica a la que tiene "derecho" el compañero, no les permite esperar una atención médica de calidad, salvo si la otorga otra mujer, con quien es más fácil establecer una identificación que favorece la relación médico-paciente. Un estudio reciente realizado en una población similar de usuarias de servicios de salud en México, sin embargo, indica que cuando -por razones económicas o culturales- la mujer enfrenta una relación desigual durante el acto clínico es muy probable que reciba una atención de baja calidad, aun cuando sea otorgada por una profesional. ${ }^{28}$

Adicionalmente, los factores socioculturales y psicológicos descritos líneas arriba, más la exposición del cuerpo durante la realización del ECM, generarán sensaciones de incomodidad en el médico, al sentir un "riesgo" en la realización del ECM, tal como se observa en la reiterada mención a la CONAMED.

Las usuarias se colocan en la posición dependiente no demandante en la interacción con el médico, y generalmente aceptan sus indicaciones sin cuestionarlas, aunque puedan hacerlo posteriormente a la ejecutora de las indicaciones (enfermera). Los médicos no "escuchan" a las usuarias. Antes de permitir que ellas 
digan el motivo de su consulta aparece un tajante "¿qué tiene?", lo que cierra la posibilidad de entablar una relación de confianza con el consultor. Es más, existe una franca predisposición a no confiar en la práctica médica institucional, la cual es vista como algo a lo que se tiene derecho, pero sin que tal derecho implique la posibilidad de obtener una franca satisfacción. La mujer tiene que aceptar al médico asignado, sin elegirlo, por lo que, de entrada, no confía en él. Estos resultados son similares a los referidos en investigaciones cualitativas realizadas sobre acceso a servicios de sa$\operatorname{lud}^{29} \mathrm{y}$, en especial, para evaluar programas de tamizaje de $\mathrm{CM}^{30,31}$

Los profesionales de la salud explican los motivos por los que no ofrecen el ECM, los cuales implican su percepción de las barreras socioculturales y psicológicas presentes en las mujeres, que se manifiestan en menor grado al haber identificación de género con las médicas pero que dificultan la solicitud del ECM a los médicos. Además, refieren sus propias barreras, donde al entrar en juego las diferencias de género se manifiestan los temores hacia la interpretación que la usuaria hace de las acciones del acto médico, las cuales pueden llegar a situaciones de queja con las instancias de auditoría médica. Las frecuentes fallas del sistema institucional, como la ausencia de enfermera, ausencia de lugar adecuado para la exploración, falta de tiempo, etcétera, pueden precipitar la interpretación inadecuada por parte de la paciente y generar al médico o a la institución problemas médico legales.

La buena relación médico-paciente favorece el ofrecimiento del examen clínico de mama y asegura la aceptación de la mujer. La calidad de la relación interpersonal en la atención médica no cumple las expectativas de buen trato que las mujeres desean recibir: ser escuchadas, informadas y atendidas por un profesional de la salud con quien puedan sentir confianza y establecer comunicación. Estas cualidades en la relación posibilitarían la autodeterminación de la usuaria frente al ECM. Desde la percepción de las usuarias los requisitos de la atención de calidad interpersonal los satisface con mayor frecuencia una médica. No obstante, el que el prestador de servicio sea mujer, como en el caso de la enfermera capacitada, facilita la aceptación del ECM pero no su demanda, ya que su papel en la provisión de servicios para la salud es subvaluado para favorecer al médico.

La falta de confianza en el médico institucional parece también una redefinición social, esto es, el médico institucional ha perdido parte de la confianza que inicialmente la sociedad había depositado en él y esto se generaliza hacia no confiar en las instituciones. El trasfondo de esta pérdida es el largo anecdotario, en el ámbito popular, de insatisfacción y abuso, que ya ha sido ampliamente informado como un fenómeno resultado de años de dominación de la práctica médica hegemónica. ${ }^{29}$ Una muestra de ello es cómo, dentro de las instituciones donde se realizó este estudio, las usuarias son caracterizadas por los médicos a partir de su ignorancia y ellos son caracterizados por las usuarias por su falta de profesionalismo y de ética, contenido que no se niega en los grupos de médicos, quienes parecen estar conscientes de lo que la sociedad opina.

Las usuarias expresan el deseo de contar preferentemente con personal femenino y privacidad para el ECM, condiciones que al parecer favorecerían que la mujer acuda a que le practiquen la exploración. Parece el encuentro de la conciencia de género, orientada a sentir el apoyo de sus iguales y a la búsqueda de atención de calidad por las mujeres y para las mujeres. Hay que cuestionar si todos los contenidos relacionados con la experiencia corporal se subordinarían a la resolución de los conflictos que impone el género del profesional, y el hecho de que la calidad de la relación interpersonal entre mujeres resulte más satisfactoria per se, mejorando la accesibilidad, independientemente de si el sistema de salud alcance o no los niveles de calidad óptima.

\section{Conclusiones}

Este trabajo presenta la importancia de la investigación en servicios de salud a través de metodología cualitativa. Los hallazgos pueden ser agrupados, al menos para esta muestra, a partir de quién proveyó la información en tres grandes rubros:

1. El profesional de la salud: a) es consciente de la necesidad de vencer algunas barreras sociales y psicológicas para que la mujer que no tiene síntomas solicite el ECM; b) identifica en su persona la presencia e importancia de estas mismas barreras, que provocan diferimiento o definitivamente ausencia de oferta del ECM; c) tiene poco interés en consolidar una buena relación médico-paciente; d) al tener poco conocimiento de la norma oficial y poca práctica en la realización del ECM, la calidad técnica del mismo es cuestionable, y e) ante estas barreras el carácter preventivo del ECM prácticamente no existe.

2. Las mujeres usuarias de los servicios de salud: a) manifiestan explícitamente barreras sociales y psicológicas que les impiden acceder al ECM; b) prefieren ser atendidas por personal de salud de su 
mismo género; c) reconocen que la desconfianza en el sistema de salud y en especial en los médicos son barreras en el acceso al ECM.

3. El sistema de salud. La implantación de los programas institucionales de detección oportuna del CM no han considerado abiertamente las condiciones socioculturales y psicológicas de las mujeres usuarias, lo que disminuye su acceso y utilización.

Estos datos permiten sugerir que las instituciones necesitan focalizar las acciones en la mejora de la calidad de la relación médico-paciente. Sólo así mejorará su imagen frente a las usuarias. Esta relación debe considerar las expectativas de las usuarias, esto es, fomentar una comunicación igualitaria basada en el respeto y el interés del médico por su "paciente", con el fin de alcanzar nuevamente la confianza de la usuaria y de que se revalore la atención recibida. Además, de manera general, las conclusiones sugieren la necesidad de continuar trabajando en la evaluación de la calidad interpersonal y técnica del ECM, área de investigación que presenta amplias oportunidades de abordaje.

\section{Referencias}

1. Secretaría de Salud. Salud México 2002. Información para la rendición de cuentas. México, DF: D irección General de Información y Evaluación del D esempeño, SSA; 2003.

2. Menck HR, Mills PK. The influence of urbanization, age, ethnicity and income on the early diagnosis of breast carcinoma: 0 pportunity for screening improvement. Cancer 2001;92(5):1299-1304.

3. Secretaría de Salud. Salud México 2001. Información para la rendición de cuentas. México, DF: Dirección General de Información y Evaluación del Desempeño, SSA; 2002.

4. Lazcano-Ponce E,Tovar-G uzmán V,Alonso-de P, Romieu I, López-

Carrillo I. Cáncer de mama. Un hilo conductor histórico, presente y futuro. Salud Publica Mex 1996; 38:139-152.

5. Bloom JR. Early detection of cancer. Psychologic and social dimensions. Cancer 1994;74(4 Suppl):1464-1473.

6. Harris JR. Lippman ME,Veronesi U, W illet W. Breast cancer (first of the three parts). Medical Progress. Review Articles. N Engl J Med 1992 July 30;37(5):319-328.

7. Secretaría de Salud. N O M 041-SSA 2-2002, para la Prevención, Diagnóstico, Tratamiento, C ontrol y Vigilancia Epidemiológica del Cáncer de Mama. México, D F: D iario 0 ficial de la Federación; Septiembre de 2003.

8. Pabón-Lasso H. Evaluación de los servicios de salud. Cali, Colombia: Universidad del Valle; 1985:47-62.

9. Frenk J. El concepto y la medición de accesibilidad. Salud Publica Mex 1985;27:438-453.

10. D onabedian A. La calidad de la atención médica. D efinición y métodos de evaluación. México, DF: La Prensa Médica Mexicana; 1984. 11. D onabedian A.The quality of medical care: How can it be assessed? JAMA 1998;260:1743-1748.
12. A rredondo A, Meléndez V. Modelos explicativos sobre la utilización de servicios de salud: revisión y análisis. Salud Publica Mex 1992;34: 36-49.

13. $N$ ash-O januga $D$, G ilbert $C$. W omen's access to health care in developing countries. Soc Sci Med 1992;35(4):613-617.

14. Schulman K, Berlin JA, Harness W, Kerner JF, Sistrunk S, Gersh J et al. The effect of race and sex on physicians' recommendations for cardiac catherization. N Engl J Med 1999;340(8):618-626.

15. Borders TF, W arner RD, Sutkin G. Satisfaction with health care and cancer screening practices among women in a largely rural region of West Texas. Prev Med 2003; 36(6):652-658.

16.Zapka JG, Berkowitz E.A qualitative study about breast cancer screening in older women: Implications for research. J Gerontol 1992 N ov;47(Spec N 0):93-100.

17. Sharpe PA. 0 Ider women and health services: Moving from ageism toward empowerment. Women Health 1995;22(3):9-19.

18. Puentes-Rosas E, Garrido F.Trato adecuado en México: respuesta a las expectativas no médicas de los usuarios de servicios de salud. Síntesis Ejecutiva. México, DF: D irección de Planeación Estratégica, Secretaría de Salud; 2002.

19. Castro R, Bronfman M.Algunos problemas no resueltos en la integración de métodos cualitativos y cuantitativos en la investigación social en salud. En: Bronfman M, Castro R, coord. Salud, cambio social y política. Perspectivas desde América Latina. México, D F: Edamex; 1999:46-64

20. Poblano-Verástegui 0, López-C arrillo L, C lemenceau RJ, LópezCervantes M.The reproducibility of breast cancer diagnosis through mammography:A pilot study in Mexico.W oman Cancer J 2000 Spring; 2(1):31-36.

21. 0 rtega $D$, López-Carrillo L, Poblano 0 . D eterminantes de la utilización de la detección oportuna del cáncer de mama.Trabajo presentado en el VII Congreso $\mathrm{N}$ acional de Investigación en Salud Pública; 1997 marzo 2 - 5; Cuernavaca, Morelos, México.

22. Secretaría de Salud, D irección General de Información y Evaluación del Desempeño. Consultas, detecciones, ingresos y casos en control de cáncer de mama, 2001. México, DF:SSA, D GIED, Subsistema de Servicios. 2002. Disponible en: http://www/ ssa.gob.mx [2003 dic 15]. 23. Instituto $\mathrm{N}$ acional de Estadística Geografía e Informática. X II Censo General de Población y Vivienda 2000. México, D F: IN EG I; 2002.

24. G laser B, Strauss A. The discovery of G rounded Theory. Strategies for qualitative research. N ueva York (N Y):A Idine De Gruyter; 1967. 25. Strauss A, C orbin J. Bases de la investigación cualitativa.Técnicas y procedimientos para desarrollar la teoría fundamentada. Antioquia: Universidad de Antioquia; 2002.

26. Basaglia F. La mujer y la locura. En: Marcos S, coord. Antipsiquiatría y política. 2a. edición. México, D F: Ed. Extemporáneos; 1984:150-171. 27. Schlesinger M.A loss of faith:The sources of reduced political legitimacy for the American medical profession. Milbank Q 2002;80(2):185-235.

28. Bronfman-PM, López MS, Magis RC, Moreno AA, Rutstein S.Atención prenatal en el primer nivel de atención: características de los proveedores que influyen en la satisfacción de las usuarias. Salud Publica Mex 2003:45:445-454

29. Puentes-Markides $C$. W omen and access to health care. Soc Sci Med 1992;35(4):619-626.

30. Mathews HF, Lannin DR, Mitchell JP. C oming to terms with advanced breast cancer: Black women's narratives from Eastern $N$ orth C arolina. Soc Sci Med 1994;38(6):789-800.

31. D anigelis N L, Robertson N L,W orden JK, Flynn BS, D orwaldt AL, A shley JA et al. Breast screening by African-A merican women: Insights from a household survey and focus groups. Am J Prev Med 1995;11(5):311-317. 